\title{
Neurotrophins Support the Development of Diverse Sensory Axon Morphologies
}

\author{
Stephen I. Lentz, ${ }^{1}$ C. Michael Knudson, ${ }^{2}$ Stanley J. Korsmeyer, ${ }^{2}$ and William D. Snider ${ }^{1}$ \\ ${ }^{1}$ Center for the Study of Nervous System Injury, Department of Neurology, and ${ }^{2}$ Department of Medicine and Pathology, \\ Howard Hughes Medical Institute, Washington University School of Medicine, St. Louis, Missouri 63110
}

\begin{abstract}
The initial outgrowth of peripheral axons in developing embryos is thought to occur independently of neurotrophins. However, the degree to which peripheral neurons can extend axons and elaborate axonal arborizations in the absence of these molecules has not been studied directly because of exquisite survival requirements for neurotrophins at early developmental stages. We show here that embryonic sensory neurons from BAX-deficient mice survived indefinitely in the absence of neurotrophins, even in highly dissociated cultures, allowing assessment of cell autonomous axon outgrowth. At embryonic day 11 (E11)-E13, stages of rapid axon growth toward targets in vivo, Bax-/- sensory neurons cultured without neurotrophins were almost invariably unipolar and extended only a rudimentary axon. Addition of neurotrophins caused outgrowth of a second
\end{abstract}

axon and a marked, dose-dependent elongation of both processes. Surprisingly, morphological responses to individual neurotrophins differed substantially. Neurotrophin-3 (NT-3) supported striking terminal arborization of subsets of Bax-/neurons, whereas NGF produced predominantly axon elongation in a different subset. We conclude that axon growth in vitro is neurotrophin dependent from the earliest stages of sensory neuron development. Furthermore, neurotrophins support the appearance of distinct axonal morphologies that characterize different sensory neuron subpopulations.

Key words: neurotrophins; nerve growth factor; neurotrophin-3; brain-derived neurotrophic factor; dorsal root ganglion; BAX; development; axon morphology
Among the most impressive effects of the neurotrophin family of neurotrophic factors is the dose-dependent increase in axon density observed in explants of peripheral ganglia from embryonic avians and mammals. Surprisingly, however, both the interpretation and implications of this phenomenon have remained unclear. An analysis of the effects of nerve growth factor (NGF) on the morphology of individual avian sensory neurons did not reveal a dose-dependent effect on axon growth at early developmental stages, suggesting that peripheral neurons elaborate axons at an optimal rate in the presence of sufficient NGF to allow survival (Scott and Davies, 1993). Dose-dependent increases in the axon density from explants may therefore be related to influences on survival rather than to direct effects of NGF on axon growth (Scott and Davies, 1993). Initial descriptions of the timing of appearance of NGF in peripheral tissues and of NGF receptors on sensory neurons in relation to the outgrowth of sensory axons in vivo also did not favor the interpretation that NGF plays any direct role in regulating growth at developmental stages in which axons are projecting toward their targets (Lumsden and Davies, 1983; Davies et al., 1987; Ernfors et al., 1992). Indeed, the favored hypothesis has been that neurotrophins act primarily to mediate branching after axons are in the vicinity of their target fields (McFarlane and Holt, 1997).

\footnotetext{
Received Aug. 3, 1998; revised Sept. 25, 1998; accepted Nov. 13, 1998.

This work was supported by National Institutes of Health Grants NS31768 and NS34448 (W.D.S.) and HD27500 (S.J.K.). S.I.L. was supported by Training Grant HL07275-18. We especially thank J. C. Harding and L. A. Worley for their expert technical assistance. We also thank P. Lampe for advice in setting up the cell cultures.

Correspondence should be addressed to Dr. William D. Snider, Center for the Study of Nervous System Injury, Department of Neurology, Box 8111, Washington University School of Medicine, 660 South Euclid Avenue, St. Louis, MO 63110. Copyright (C) 1999 Society for Neuroscience $\quad 0270-6474 / 99 / 191038-11 \$ 05.00 / 0$
}

Several recent observations, however, are consistent with the idea that neurotrophins might regulate axon growth even at early developmental stages. For example, dorsal root ganglia (DRG) neurons express neurotrophin receptors and require neurotrophins for survival as early as embryonic day 11.5 (E11.5), indicating a capacity to respond to these molecules during early stages of axon growth (Fariñas et al., 1996; White et al., 1996). Furthermore, neurotrophin-3 (NT-3) is synthesized in mesenchyme along the pathways of developing sensory and sympathetic axon projections as early as E10, consistent with the idea that NT-3 could influence early axon growth of several classes of peripheral neurons (Fariñas et al., 1996; Verdi et al., 1996; White et al., 1996; Wilkinson et al., 1996). Abnormalities in axon projections of vestibular and cochlear ganglia have been documented in BDNFand NT-3- (and trkB- and trkC-) null mice (Ernfors et al., 1995; Schimmang et al., 1995; Fritzsch et al., 1997), and the extension of sympathetic axons to distal targets is deficient in trkA nulls (Fagan et al., 1996). However, it has been difficult to separate regulation of axon growth from regulation of survival in the setting of neurotrophin and/or trk deficiency. Interestingly, FGF2 and FGF receptor signaling significantly influences the rate of extension of retinal ganglion cell axons along the optic tract in Xenopus (McFarlane et al., 1995). Whether FGF2 or any other neurotrophic factor has a role in regulating axon extension in mammals is unknown (for review, see McFarlane and Holt, 1997).

A major difficulty in studying the role of neurotrophins in axon growth at early developmental stages has been the absolute survival requirement of many classes of peripheral neurons on one or more neurotrophin family members. Thus, it has not been possible to examine axon outgrowth directly in the absence of these molecules either in vivo or in vitro. Recently, however, 
progress in our understanding of apoptosis has led to the discovery of conditions in which peripheral neurons can survive in vitro and in vivo in the absence of exogenous neurotrophins (for review, see Johnson et al., 1996). For example, in mice that are null for the apoptosis regulator BAX, sympathetic ganglion neurons survive indefinitely in vitro in the absence of NGF, and neonatal motor neurons survive axotomy in vivo (Deckwerth et al., 1996). Studies of naturally occurring cell death in Bax nulls suggest that many classes of peripheral neurons including DRG neurons are similarly regulated (White et al., 1998). Importantly, Bax-/- mice survive into adulthood, do not have gross abnormalities in either the peripheral nervous system (PNS) or the CNS, and have increased numbers of axons in peripheral and optic nerves (White et al., 1998). These findings demonstrate that axon growth and connectivity are not profoundly affected by the absence of this molecule.

To study the dependence of axonal morphology on neurotrophins at developmental stages at which peripheral neurons require these molecules for survival in vivo, we have cultured sensory neurons of the DRG from E11-E13 Bax-/- mice. The Bax null mutation allows sensory neurons to survive in highly dissociated cultures in which neurons are virtually devoid of any trophic influences from non-neuronal cells and axons of individual neurons can be traced in their entirety. Our findings demonstrate that sensory neurons invariably extend only a single rudimentary axon in the absence of these molecules. Unexpectedly, we have found that NGF, NT-3, and BDNF each support a distinct axonal morphological response from a subset of sensory neurons. We suggest that neurotrophins support the massive elongation necessary for peripheral axons to keep pace with growth of the embryo and that these molecules support the development of diverse sensory axon morphologies.

\section{MATERIALS AND METHODS}

Animals. The Animal Studies Committee of Washington University approved all experimental procedures involving animals. Bax $+/-$ male and female mice were bred to produce Bax+/+, Bax+/-, and Bax-/offspring. The plug date was considered E0. Whole embryos were dissected from sodium pentobarbitol-overdosed mothers under sterile conditions and were collected in ice-cold L15 medium supplemented with 5\% heat-inactivated (HI) horse serum. Embryos were harvested on E11-E13, and the developmental stage was verified by crown rump measurements. Tails were used as a source of DNA to determine the genotype of individual embryos by PCR. Sequences for the PCR primers have been published (White et al., 1998).

Dissociated cell cultures. A modification of previously published methods (Eichler and Rich, 1989) was used to prepare cultures of dissociated DRG neurons. Importantly, separate cultures were established for each embryo. Embryos were subsequently genotyped as described above. Ganglia from the entire rostrocaudal extent of the spinal cord were dissected and collected in ice-cold L15 media supplemented with 5\% HI horse serum. Cells were dissociated enzymatically with $1 \mathrm{mg} / \mathrm{ml}$ collagenase A (Boehringer Mannheim, Indianapolis, IN) at $37^{\circ} \mathrm{C}$ for $15 \mathrm{~min}$ followed by $0.05 \%$ trypsin and $0.02 \%$ EDTA at $37^{\circ} \mathrm{C}$ for $7 \mathrm{~min}$. Trypsin was inactivated with 4 vol of Minimal Essential Medium (MEM; Life Technologies, Gaithersburg, MD) containing 5\% HI fetal bovine serum (Summit, Fort Collins, CO), and ganglia were collected by brief centrifugation. MEM and trypsin were removed, and ganglia were resuspended in MEM containing 5\% HI fetal bovine serum, $2 \mathrm{mM}$ L-glutamine, and $1 \times$ penicillin/streptomycin. Cells were then mechanically dissociated by trituration through full bore-sized and one-third bore-sized Pasteur pipettes. Non-neuronal cells were eliminated with 5-fluoro-2'deoxyuridine (Sigma, St. Louis, MO) added to the medium at a final concentration of $10 \mu \mathrm{M}$. Total cell counts and viable cell numbers were determined by trypan blue exclusion and a hemacytometer. Dissociated cells were plated on autoclaved glass coverslips (Thomas Scientific) coated overnight with a mixture of poly-D-lysine $(0.1 \mathrm{mg} / \mathrm{ml}$; Sigma $)$ and laminin (4 ng/ml; Collaborative Biomedical Products) in 24 well sterile culture plates (Fisher Scientific, Houston, TX) at 500 or 2000 cells per well. Some cultures were supplemented with NGF, NT-3, BDNF, or NT-4 (each at $50 \mathrm{ng} / \mathrm{ml}$, unless otherwise noted) at the time of plating. Sister cultures were maintained without added neurotrophins. Each experiment was repeated with embryos from three to five separate matings.

The percentage of Bax-/- neurons that survive in the absence of neurotrophins was quantified. The initial number of neurons was determined by counting phase-bright cells $2 \mathrm{hr}$ after plating. The numbers of phase-bright neurons after 24 and $72 \mathrm{hr}$ were compared with the initial number of neurons. Approximately 90 and $60 \%$ of Bax-/- neurons survived in the absence of neurotrophins after 24 and $72 \mathrm{hr}$, respectively. In contrast, $<5 \%$ of $\mathrm{Bax}+/+$ neurons survived after $24 \mathrm{hr}$, and no neurons were present after $72 \mathrm{hr}$.

Immunocytochemistry. Cultures were maintained for $3 \mathrm{~d}$ (unless otherwise noted) and then fixed in $4 \%$ paraformaldehyde and $0.025 \%$ glutaraldehyde in PBS for $30 \mathrm{~min}$ at room temperature. Cell morphology was visualized using a monoclonal antibody directed against phosphorylated neurofilament $\mathrm{H}$ (NFH) and M (NFM) (SMI 31; Sternberger Monoclonals Inc., Baltimore, MD). For immunohistochemistry, cultures were blocked in Superblock buffer (Pierce, Rockford, IL) with $1 \%$ porcine gelatin, $0.2 \%$ Triton $\mathrm{X}-100$, and $1.5 \%$ goat serum for $30 \mathrm{~min}$ at room temperature. Primary antibody was added at a concentration of 1:1000 and incubated overnight at $4^{\circ} \mathrm{C}$. The signal was amplified with the Vectastain $\mathrm{ABC}$ kit (Vector Laboratories, Burlingame, CA) following the manufacturer's protocols and was visualized with a solution containing $500 \mathrm{ng} / \mathrm{ml}$ diaminobenzidine tetrahydrochloride.

Quantification of axon number and soma size. Coverslips were mounted on slides with DPX, and neurons were viewed in bright field on a Nikon Optiphot microscope with a $40 \times$ objective. Randomly selected neurons from the bottom one-third of each coverslip were traced with a camera lucida. Except for Bax+/+ neurons treated with NT-3, 50 neurons were drawn from each of six embryos from three separate culture experiments. Thus, a total of 300 neurons was analyzed for each experimental condition. For Bax $+/+$ neurons treated with NT-3, only 145 neurons were drawn because of the low number of surviving neurons in this condition. Axons $>50 \mu \mathrm{m}$ in length were scored. The percentage of neurons with one or two or more axons was calculated for each animal. The drawings were scanned into a Macintosh computer, and soma areas were quantitated with National Institutes of Health Image version 1.61 software.

Quantification of total axon length and number of branch points. For quantification of total axon length, neurons from very low density cultures (500 cells per well) were viewed in bright field with a $10 \times$ objective. Only neurons completely isolated from neighboring neurons were used. For each experimental condition, camera lucida drawings were made of a total of 50 neurons from six to seven embryos from three to four separate culture experiments. The drawings were scanned into a Macintosh computer, and axon lengths were quantified using National Institutes of Health Image version 1.61 software. Total axon length was determined by summing the lengths of all axons for each neuron. The number of branch points per neuron was also determined from this population. A branch point was counted if the axon formed a branch that continued a distance $\geq 25 \mu \mathrm{m}$.

Statistical analysis. Data from individual embryos were pooled and used for statistical analyses. Overall significant differences between conditions were determined by one-way ANOVA. Post hoc comparisons were done with the Tukey test. The data presented in the figures and the text represent means and SEMs from five to seven embryos.

\section{RESULTS}

\section{Sensory axon outgrowth and fasciculation are dependent on neurotrophins}

Figure $1 a$ shows the typical appearance of sensory neurons in dissociated cultures (2000 cells plated per well of a 24 well plate) from wild-type $(\mathrm{Bax}+/+)$ mice at E13 cultured in the presence of NGF. Neuronal somata and axons were stained with SMI 31, a monoclonal antibody to phosphorylated epitopes of NFM and NFH. Cultures from Bax-/- mice in the absence of NGF (Fig. $1 b$ ) looked strikingly different with much less extensive neurite outgrowth. As shown in Figure 1c, culturing sensory neurons from Bax-/- mice with $50 \mathrm{ng} / \mathrm{ml} \mathrm{NGF}$ restored neurite out- 


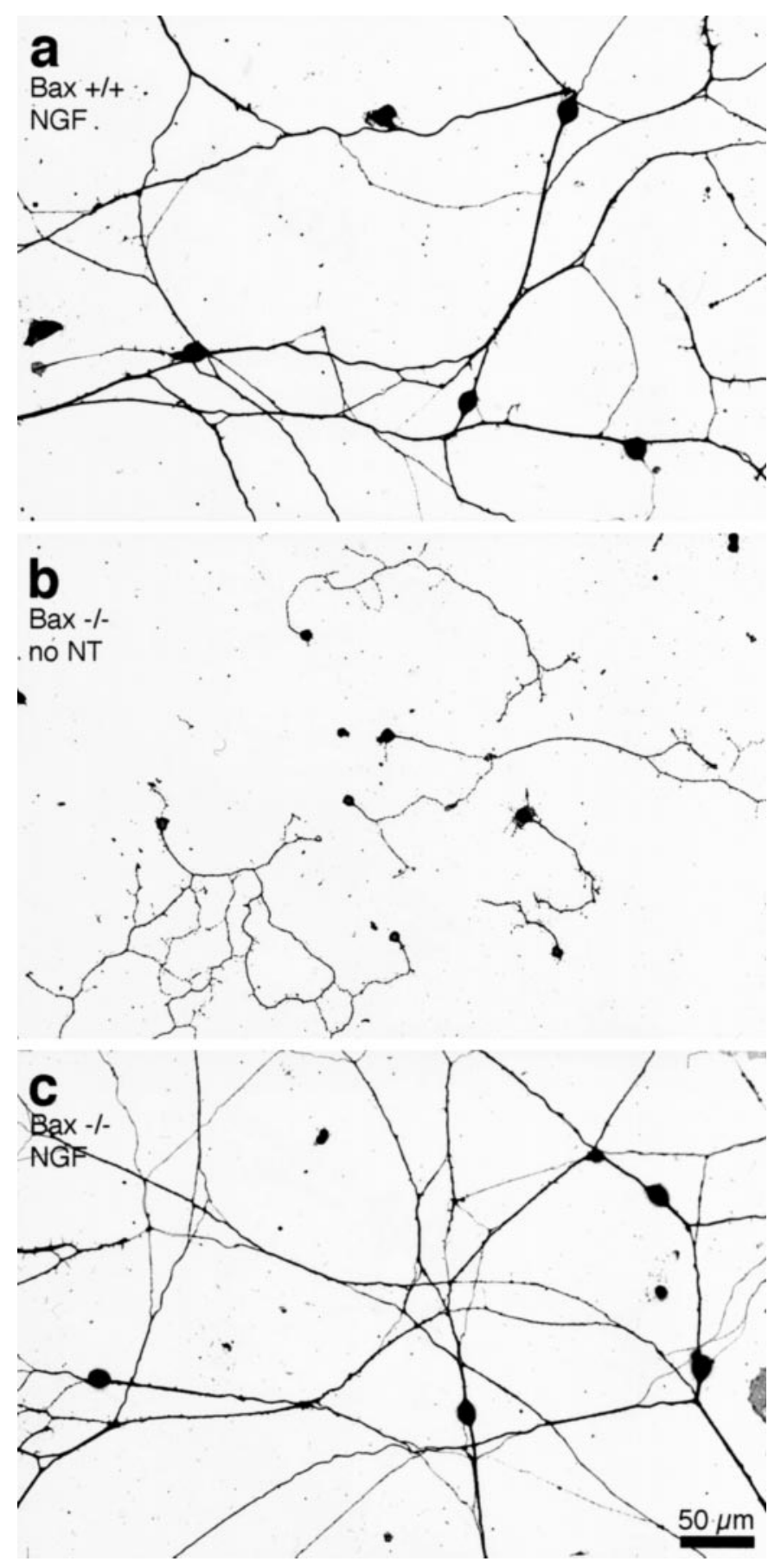

Figure 1. Sensory axon growth is rudimentary in the absence of neurotrophins. Photomicrographs of neuronal cultures plated at moderate densities (2000 neurons per well) and grown for $72 \mathrm{hr}$ in the presence or absence of $50 \mathrm{ng} / \mathrm{ml} \mathrm{NGF}$ are shown. $a$, Neurons from Bax $+/+$ mice extend long, fasciculated axons in the presence of $N G F$. $b$, Neurons from Bax $-/-$ mice in the absence of neurotrophins (no NT) extend short, branched axons that do not form fascicles. $c$, In the presence of $N G F$, cultures from $\mathrm{Bax}-/-$ mice look indistinguishable from controls.

growth to a pattern that was indistinguishable from that of controls.

Obvious differences in the extent of fasciculation between treated and untreated cultures were also apparent in every experiment. In $\mathrm{Bax}+/+$ mice in the presence of NGF, fasciculation of almost all major axon trunks was readily apparent (Fig. 1a). In contrast, Bax-/- DRG cultures exhibited little axon fasciculation in the absence of neurotrophins (Fig. 1b). Under neurotrophindeficient conditions, axons apparently preferred the laminin substratum for growth compared with neighboring axons. Treatment of the Bax-/- cultures with NGF restored fasciculation and led to an appearance virtually identical to that of wild-type cultures (Fig. 1c).

\section{Sensory neurons are unipolar in the absence of neurotrophins}

Morphological responses of $\mathrm{Bax}-/-$ neurons in the presence and absence of neurotrophins were characterized in detail at E13. At high and moderate densities, it was difficult to determine the parameters of axon growth and branching that were affected by NGF. We therefore prepared highly dissociated cultures (500 neurons plated per well). Of note is that these cultures prepared from E13 mice were almost devoid of non-neuronal cells (see Materials and Methods).

Analysis of individual neurons in these low-density cultures revealed that in the absence of neurotrophins, neurons from Bax-/- mice were almost invariably unipolar and extended short, branched axons (Fig. 2a). When Bax-/- neurons were grown in the presence of NGF, a subpopulation of neurons responded by assuming a bipolar configuration and sending out long axons (Fig. 2b). Treatment with NT-3 also resulted in bipolar morphology with extensive axon outgrowth as well as terminal branching (see below) in a subpopulation of neurons (Fig. 2c).

The number of unipolar, bipolar, and multipolar (three or more axons) neurons at E13 was quantified in low- and moderatedensity cultures. The histograms in Figure $2 d$ show that $>90 \%$ of Bax $-/-$ neurons cultured without neurotrophins were unipolar, $<10 \%$ were bipolar, and there were no neurons in an extensive sample that possessed three or more axons. When neurons from Bax-/- mice were cultured for $72 \mathrm{hr}$ in the presence of NGF, $55 \%$ of the neurons had two or more axons. Note that this does not approach the figure for $\mathrm{Bax}+/+$ neurons in which almost $90 \%$ had two or more axons when data from the low- and moderatedensity cultures were pooled. Presumably all classes of neurons survive in Bax-/- cultures, i.e., those that normally respond to NT-3 and potentially other growth factors in addition to NGF. Thus, it is not surprising that a substantial percentage of neurons in these cultures did not respond to NGF with growth of a second axon.

Results with NT-3 were qualitatively similar (Fig. 2e). Thus, $\sim 30 \%$ of $\mathrm{Bax}-/-$ neurons in NT-3-treated cultures had two or more axons compared with $<10 \%$ for untreated neurons. Again, almost $70 \%$ of neurons that remained unipolar even in the presence of NT-3 presumably represented an NT-3-unresponsive population that had been saved from apoptosis in the Bax-/mice. The addition of both neurotrophins together was additive and produced a response in $83 \%$ of the cells ( $n=4$ embryos).

The effect of neurotrophins on soma size was also quantified. The addition of NGF resulted in a modest increase in soma size among the responsive Bax-/- neurons compared with Bax-/neurons cultured in the absence of neurotrophins [mean soma area, $206.3 \pm 8.8 \mu \mathrm{m}^{2}( \pm \mathrm{SEM})(n=6)$ compared with $157.1 \pm$ $\left.4.8 \mu \mathrm{m}^{2}(n=6)\right]$. As expected because many NT-3-responsive neurons are proprioceptors, soma sizes of responsive Bax-/neurons in NT-3-treated cultures were even larger (mean soma area, $\left.239.4 \pm 14.5 \mu \mathrm{m}^{2} ; n=6\right)$. 

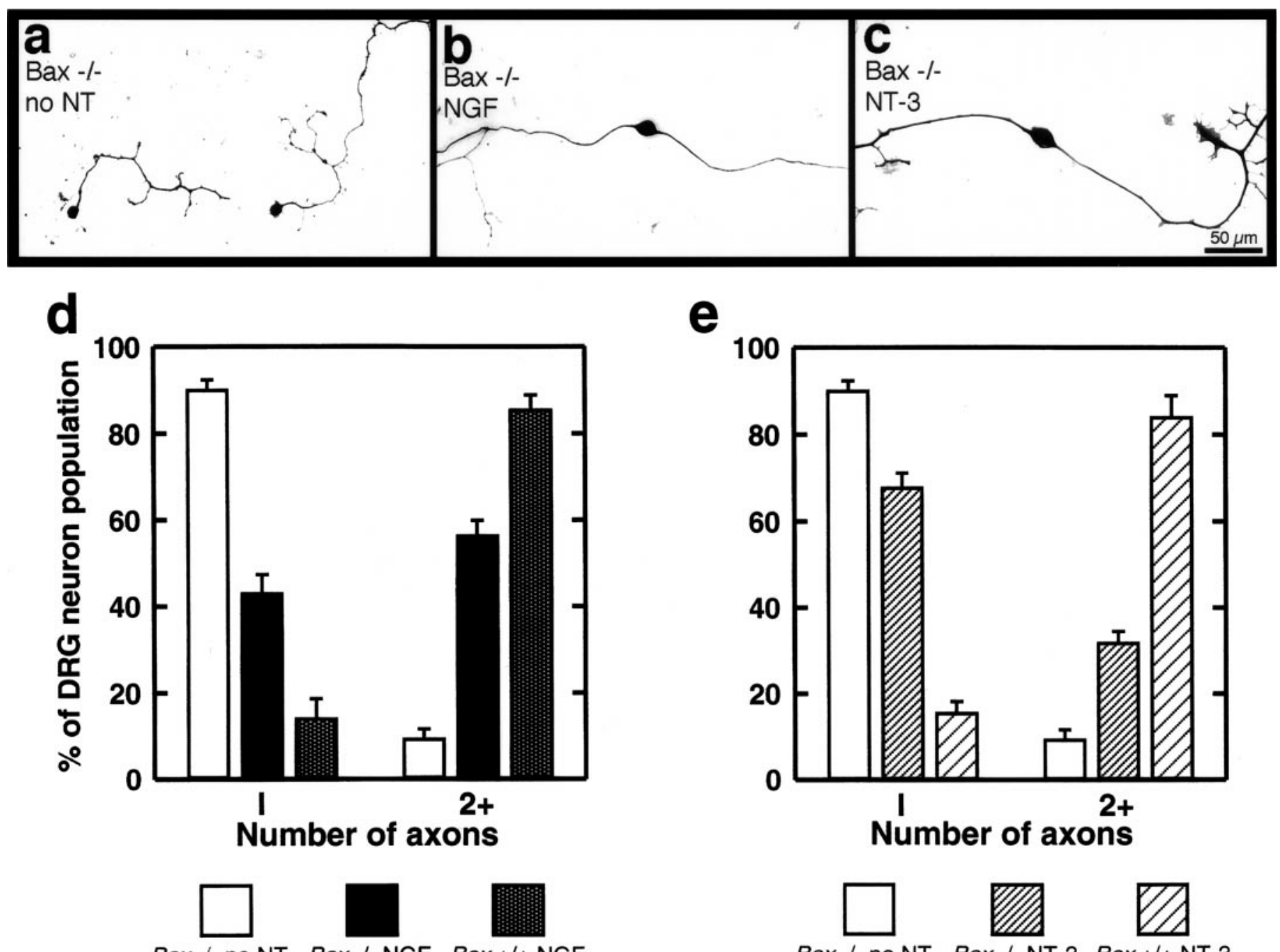

Bax $-/-$ no NT Bax $/$ - NGF Bax $+/+$ NGF
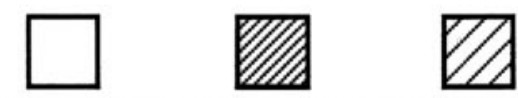

Bax $-/-$ no NT Bax $-/-$ NT-3 Bax $+/+$ NT-3

Figure 2. Quantification of neurotrophin responses. $a-c$, Photomicrographs show the typical pattern of axon extension from representative Bax-/neurons grown for $72 \mathrm{hr}$ in vitro without neurotrophins $(a)$ or in the presence of $50 \mathrm{ng} / \mathrm{ml} N G F(b)$ or $N T-3(c)$. $d, e$, Bar graphs show the percentage of neurons $( \pm$ SEM) with one or two or more axons from Bax $-1-$ mice and Bax $+/+$ littermates. Under these culture conditions, almost all neurons from Bax $+/+$ mice in the presence of either $N G F$ or $N T-3$ were bipolar (stippled bar in $d$ and wide-hatched bar in $e$, respectively). In contrast, in the absence of added neurotrophins (no NT), $90 \%$ of Bax-1- neurons were unipolar (open bars in $d, e$ ). Addition of either NGF (solid bars in $d$ ) or NT-3 ( fine-hatched bars in $e$ ) resulted in a substantial fraction of $B a x-/-$ neurons having two or more axons. Differences in percentages of neurons with a single axon between the no neurotrophin group and the groups treated with $N G F$ or $N T-3$ were highly significant ( $p<0.001 ; n=6$ embryos per group).

\section{Axon extension is rudimentary in the absence of neurotrophins}

Figure 3 shows camera lucida tracings of neurons from Bax-/mice maintained for $3 \mathrm{~d}$ without neurotrophins and of neurons from Bax-/- or wild-type mice maintained for $3 \mathrm{~d}$ in the presence of NGF or NT-3. The neurons presented were selected by ranking the neurons according to total axon length and selecting every fifth or tenth neuron. Thus, neurons presented in Figure 3 accurately reflect the differences that were observed across the entire population of neurons.

A dramatic effect of NGF on the length of axons after $3 \mathrm{~d}$ was readily apparent from the tracings (Fig. $3 b, d$ ). The appearance of Bax-1- neurons cultured without neurotrophins was very uniform (Fig. 3a). The neurons invariably had short, branched axons with total lengths of $\sim 500 \mu \mathrm{m}$ (Fig. $4 a$ ). In contrast, neurons from Bax $+/+$ mice treated with NGF were six times longer and showed little branching except at the distal tips of the axons (Figs. 3d, 4a).

Bax-/- neurons treated with NGF fell into two distinct populations (Figs. 3b, 4a). Thus, bipolar neurons extended long axons in response to NGF, similar in appearance and total length to their wild-type counterparts. In contrast unipolar neurons extended only rudimentary axons. These presumably represent neurons that are unresponsive to NGF but survive because of the Bax null mutation.

It is worthwhile noting that treatment with NGF did not completely restore to normal the length of the axons from Bax-null neurons. Thus after $72 \mathrm{hr}$, the total length of axons from bipolar Bax-null neurons was $\sim 65 \%$ of axon length in Bax $+/+$ cultures (Fig. 4a). This may indicate a minor role for Bax in regulating axon growth (see below) or alternatively may be a result of inclusion of a few Bax-/- neurons that may be bipolar but 


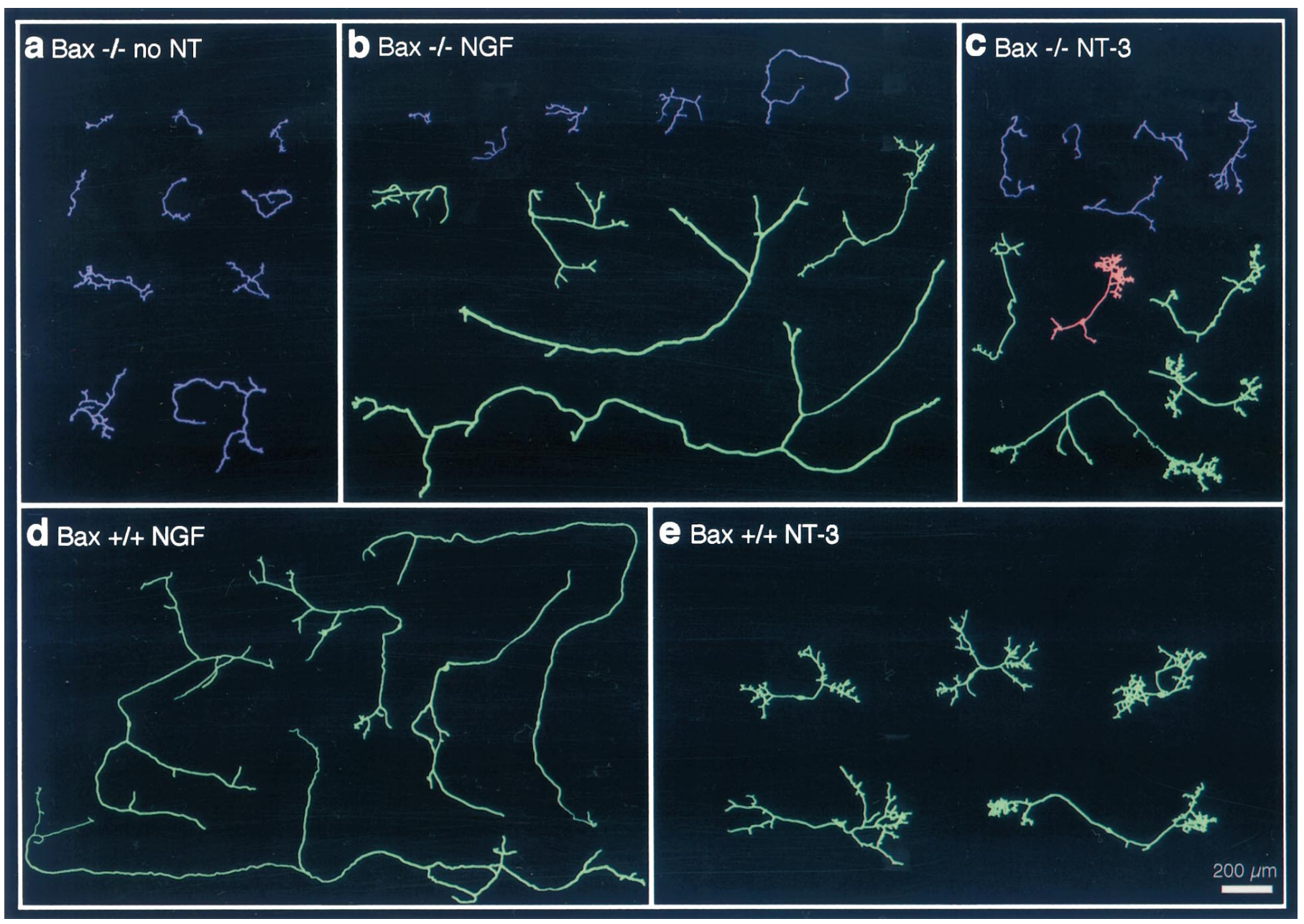

Figure 3. Morphological responses to NGF and NT-3. Camera lucida drawings of isolated DRG neurons from Bax $-/-$ and Bax $+/+$ cultures after 72 $\mathrm{hr}$ in the absence of neurotrophins or in the presence of $50 \mathrm{ng} / \mathrm{ml} \mathrm{NGF}$ or NT-3 are shown. Neurons with one (blue) or two or more (green and pink, respectively) axons were considered separately. Axons were arranged in order of increasing total length. A periodic sample of the entire population is shown for each condition. $a, B a x-/-$ neurons cultured without neurotrophins (no NT) invariably had short highly branched axons. $b$, Addition of $N G F$ resulted in the appearance of a subpopulation of $B a x-/-$ neurons with much longer axons. $c$, Addition of NT-3 also resulted in extensive axon elongation, but note that the appearance of $N T$-3-responsive neurons differed markedly from the appearance of those that responded to $N G F$. $d, e$, Representative $B a x+/+$ neurons from highly dissociated cultures in the presence of $N G F(d)$ or NT-3 $(e)$ are shown.

unresponsive to NGF. Indeed bipolar Bax-/- neurons in the absence of NGF had a total axon length of only $598.9 \pm 66.3 \mu \mathrm{m}$ $( \pm \mathrm{SEM} ; n=4)$. Unipolar Bax $+/+$ neurons in the presence of NGF were not distinguishable from bipolar $\mathrm{Bax}+/+$ neurons and had a total axon length of $2214.7 \pm 348.7 \mu \mathrm{m}(n=4)$.

Responses of $\mathrm{Bax}-/-$ neurons to NT-3 were in some ways similar, although important differences were apparent. Thus, neurons from $\mathrm{Bax}-1-$ mice that were responsive to NT-3 (i.e., neurons with two or more axons) had 3.5-fold greater total axon length than did unipolar, unresponsive neurons or neurons grown for $72 \mathrm{hr}$ in the absence of neurotrophins (Figs. 3a,c, 4b). The total axon length of Bax-/- neurons in cultures treated with NT-3 was not significantly different from the total axon length of neurons from wild-type mice (Figs. $3 c, e, 4 b$ ). Interestingly, however, axons of $\mathrm{Bax}-/-$ neurons treated with NT-3 did not achieve the axon length of neurons grown in the presence of NGF. Another very striking feature of the NT-3-treated neurons was the high degree of axon branching, which made these neurons look obviously different from their NGF-treated counterparts (see below). Again the few unipolar neurons in the Bax+/+ cultures treated with NT-3 had lengths indistinguishable from that of the bipolar neurons under this condition $(1847.8 \pm 365.9$ $\mu \mathrm{m} ; n=4)$.

It has been reported for avian neurons that at early developmental stages, concentrations of NGF sufficient to allow survival support maximum neurite outgrowth (Scott and Davies, 1993). Under our culture conditions, however, mouse sensory neurons exhibited a clear increase in axon length in association with increasing concentrations of NGF. Thus, over a $24 \mathrm{hr}$ period in culture, axon length increased almost fourfold in the concentration range of $50 \mathrm{pg} / \mathrm{ml}$ to $50 \mathrm{ng} / \mathrm{ml} \mathrm{NGF}$ (Fig. $4 c$ ). The reason why our result is different from that reported by Scott and Davies (1993) is not immediately apparent, although we note that avian trigeminal neurons were used in their study and neurons were cultured for different lengths of time.

Substantial axon growth toward peripheral targets in vivo occurs before E13. To assess neurotrophin-independent axon growth at earlier stages, we cultured sensory neurons from E11 and E12 Bax $-/$ - mice. Even at these ages, Bax - / - sensory axon extension was rudimentary in the absence of neurotrophins. In- 

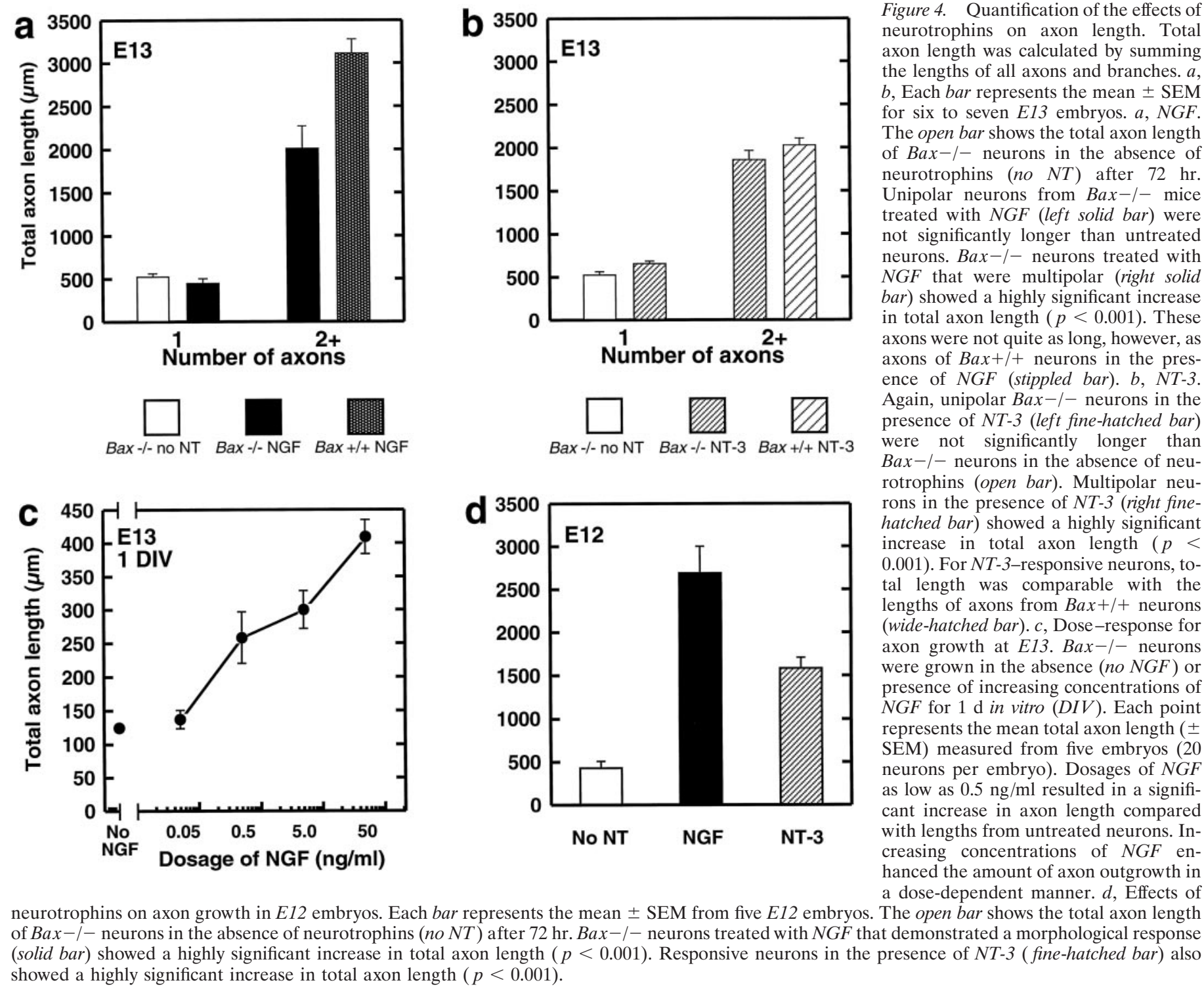

neurotrophins on axon growth in $E 12$ embryos. Each bar represents the mean + SEM from five $E 12$ embryos. os. The open bar shows the total axon length

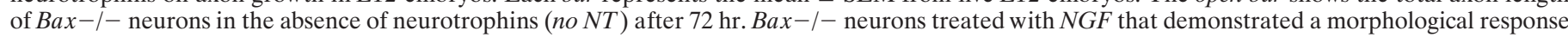

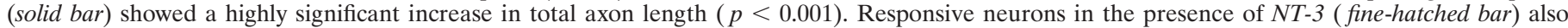
showed a highly significant increase in total axon length $(p<0.001)$. deed, the responses to added neurotrophins were even more impressive than were those of E13 neurons (Fig. 4d), with NGF inducing a sixfold increase in axon length.

Interestingly, at E12, there was a reversal in percentages of neurons responding to individual neurotrophin family members with $\sim 60 \%$ of neurons responding to NT-3 whereas only $40 \%$ responded to NGF. This may reflect the fact that trkC-expressing neurons are generated somewhat earlier than are trkA-expressing neurons. It should be noted that many trkA-expressing neurons also express trkC at this age (Ernfors et al., 1992; White et al., 1996; see also Buchman and Davies, 1993). However, increasing the NT-3 concentration had no effect on the percentage of cells exhibiting a morphological response, perhaps making activation via trkA a less likely possibility.

At E12, few Bax+/+ neurons under these highly dissociated conditions survived for $72 \mathrm{hr}$ even in the presence of neurotrophins. At E11, few neurons of any genotype survived in any condition. The few Bax-/- neurons surviving at E11 in the absence of neurotrophins exhibited almost no axon outgrowth, whereas at least some neurons extended axons in the presence of neurotrophins at this age (data not shown).
Figure 4. Quantification of the effects of neurotrophins on axon length. Total axon length was calculated by summing the lengths of all axons and branches. $a$, $b$, Each bar represents the mean \pm SEM for six to seven $E 13$ embryos. $a, N G F$. The open bar shows the total axon length of Bax-/- neurons in the absence of neurotrophins (no NT) after $72 \mathrm{hr}$. Unipolar neurons from Bax-/- mice treated with NGF (left solid bar) were not significantly longer than untreated neurons. Bax $-/-$ neurons treated with $N G F$ that were multipolar (right solid bar) showed a highly significant increase in total axon length $(p<0.001)$. These axons were not quite as long, however, as axons of $B a x+1+$ neurons in the presence of NGF (stippled bar). b, NT-3. Again, unipolar Bax-/- neurons in the presence of NT-3 (left fine-hatched bar) were not significantly longer than Bax-/- neurons in the absence of neurotrophins (open bar). Multipolar neurons in the presence of NT-3 (right finehatched bar) showed a highly significant increase in total axon length $(p<$ $0.001)$. For $N T$-3-responsive neurons, total length was comparable with the lengths of axons from Bax $+/+$ neurons (wide-hatched bar). $c$, Dose-response for axon growth at E13. Bax-/- neurons were grown in the absence (no NGF) or presence of increasing concentrations of $N G F$ for $1 \mathrm{~d}$ in vitro $(D I V)$. Each point represents the mean total axon length ( \pm SEM) measured from five embryos (20 neurons per embryo). Dosages of $N G F$ as low as $0.5 \mathrm{ng} / \mathrm{ml}$ resulted in a significant increase in axon length compared with lengths from untreated neurons. Increasing concentrations of $N G F$ enhanced the amount of axon outgrowth in a dose-dependent manner. $d$, Effects of presence of NT-3 ( fine-hatched bar) also
part demonstrated a morphological response

\section{Differing effects of NGF, NT-3, and BDNF on axon branching}

Representative appearances of axons in the various conditions at lower (left-hand panels) and higher (middle and right-hand panels) power are shown in Figure 5. Sensory neurons from $\mathrm{Bax}-/-$ mice exhibited a stereotypical pattern of small, thin branches along the entire length or at least the distal one-half of the axon (Fig. $5 a-c$ ). In contrast, in both wild-type and Bax-/- mice treated with NGF, neurons were devoid of branches along most of the length of the axon (Fig. 5d). However, in the most distal portions of axons, branches of NGF-treated neurons were longer and thicker than were the axons of neurons cultured without neurotrophins (Fig. 5e,f). These observations suggest that a primary effect of NGF at early developmental stages is to enhance interstitial lengthening and to suppress branching along the major portion of the axon with enhancement of branching only in distal axonal segments.

In cultures treated with NT-3, the pattern differed markedly in that terminal branching was far more extensive. Thus, NT-3responsive neurons from both $\mathrm{Bax}+/+$ and $\mathrm{Bax}-/-$ cultures 


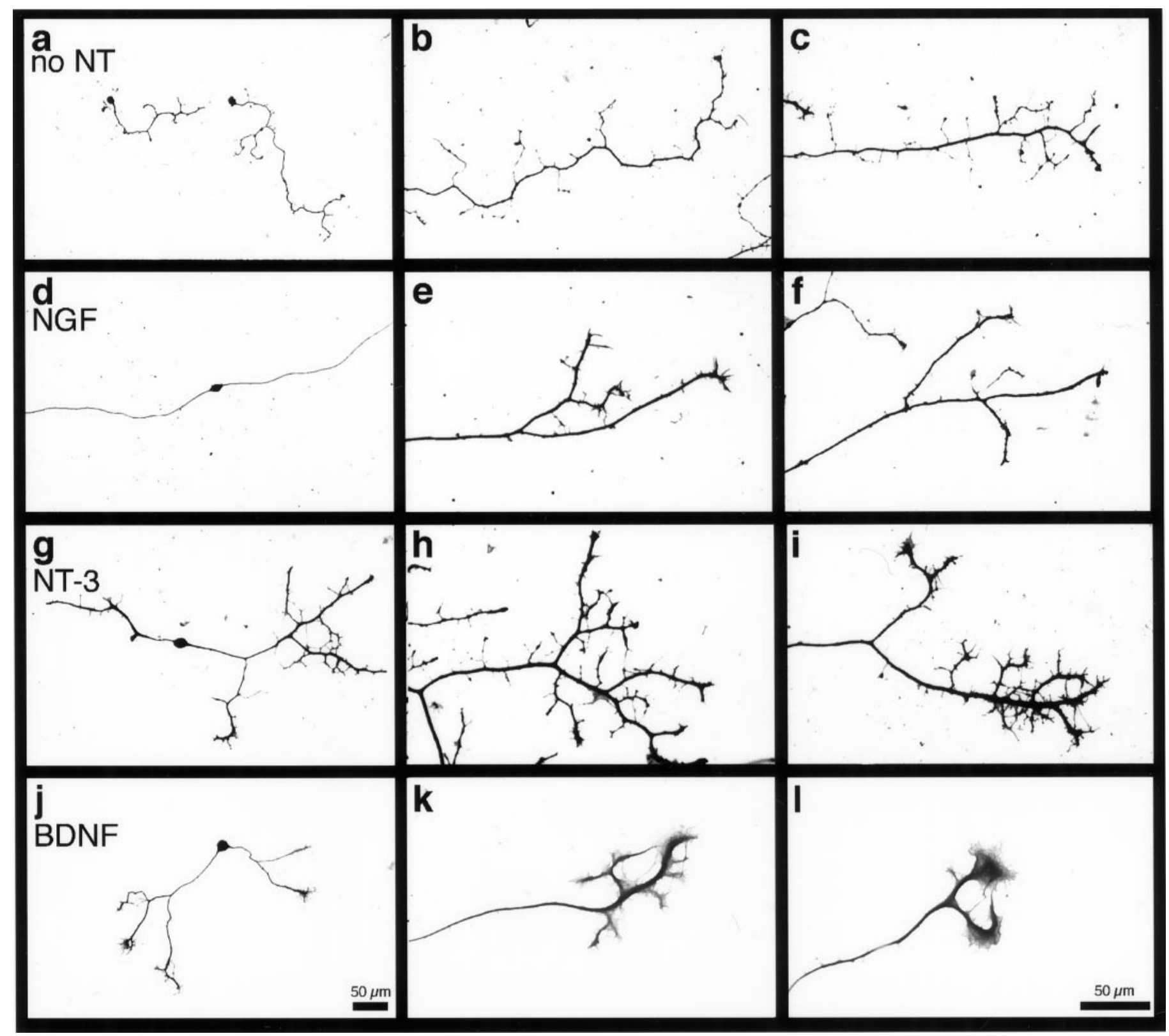

Figure 5. $N G F, N T-3$, and $B D N F$ mediate different morphological responses. $a, d, g, j$, Low-power photomicrographs (scale bar in $j$ ). $b, c, e, f, h, i, k$, $l$, High-power photomicrographs (scale bar in $l$ ). For visualizing morphologies of individual cells, neurons from Bax- $/-$ mice were plated at low density (500 neurons per well) and grown for $72 \mathrm{hr}$ in vitro without neurotrophins or in the presence of $50 \mathrm{ng} / \mathrm{ml} N G F, N T-3$, or $B D N F$. $a-c$, Representative $B a x-/-$ neurons cultured in the absence of neurotrophins (no NT). The neurons were unipolar and extended numerous short branches from the primary axon along its entire length. $d-f$, Typical $N G F$-responsive Bax-/- neurons cultured in the presence of $N G F$. These neurons were bipolar and extended long, relatively unbranched axons. $N G F$ treatment suppressed the extension of short branches close to the cell soma. Typically, a few thick caliber branches were present along the distal portion of the axon. $g-i$, Typical NT-3-responsive Bax-/- neurons in the presence of NT-3. NT-3 also appeared to suppress branching near the soma, although the primary axons of neurons treated with $N T-3$ were shorter than were those of $N G F$-treated neurons. $N T$-3-responsive neurons almost invariably exhibited elaborate branching at the distal ends of their axons. $j-l$, Representative Bax-/- neurons depicting the characteristic morphological appearance of a subset of neurons that respond to $B D N F$. Note the prominent lathellipodia.

usually possessed numerous thick branches with extensive tertiary arborization emerging from the distal segments of their axons (Fig. $5 g-i$ ). This branching response is perhaps surprising because axons are elongating toward targets and surrounded by NT-3 synthesized in mesenchyme at this age. However, this branching pattern was also prominent in most neurons that responded to NT-3 even at E12.

The effect of NGF and NT-3 on branching was quantified (Fig. $6)$. Most branches of $B a x-/-$ neurons were very short and there- fore did not extend long enough for their origins to be considered branch points (see Materials and Methods). Thus, E13 Bax-/neurons grown in the absence of neurotrophins for $72 \mathrm{hr}$ had relatively few branch points per neuron. The presence of NGF caused a moderate increase in the number of branch points per neuron. Treatment of cultures with NT-3 resulted in a very substantial increase in the number of branch points. The difference in axon branching between NGF- and NT-3-treated neurons was also apparent when total axon length was taken into 


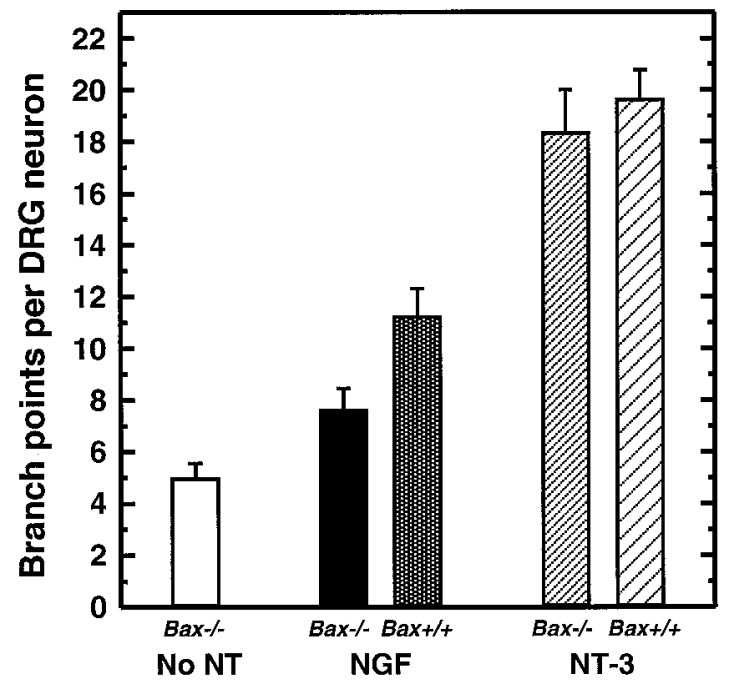

Figure 6. NGF and NT-3 differentially regulate the extent of axonal branching. Bars show the mean number of branch points per E13 DRG neuron ( $\pm \mathrm{SEM})$ for six embryos. In the absence of neurotrophins (no $N T), B a x-1-$ neurons had approximately five branch points per neuron after $72 \mathrm{hr}$ in vitro. Treatment of Bax-/- and Bax+/+ neurons with NGF resulted in a slight increase in the number of branch points per neuron. The presence of NT-3 resulted in a highly significant increase in the number of branch points per neuron $(p<0.001)$.

account. NGF-responsive neurons had $4.0 \pm 0.5( \pm \mathrm{SEM} ; n=6)$ whereas NT-3-responsive neurons had $10.6 \pm 0.8(n=6)$ branch points per millimeter of total axon length.

When both NGF and NT-3 were added to E13 cultures, cells exhibiting both morphological responses were observed as predicted if NGF and NT-3 act on different populations. Under these conditions, $54 \%$ of neurons exhibited a clear NGF-type morphology, and $13 \%$ had a clear NT-3-like morphology. Sixteen percent could not be confidently classified, possibly because these morphological criteria are not absolute or because a small subpopulation responds to both factors.

BDNF supported a branching response in some ways similar to that induced by NT-3 (Fig. $5 j$ ). At E12, $40 \%$ of the population of Bax-/- neurons responded to BDNF with a change in axon morphology. In addition to branching, however, BDNF induced a marked lamellipodial response along distal axon shafts of $\sim 30 \%$ of the responsive population at E12 (Fig. 5k,l). The significance of this characteristic morphology is unknown. Interestingly, $B a x+/+$ neurons did not survive in the presence of BDNF alone.

Finally NT-4 supported responses of fewer neurons at these ages $(\sim 20 \%)$. Morphological responses fell into both the NGFlike and NT-3-like category (data not shown).

\section{DISCUSSION}

Elimination of BAX has allowed neurotrophin regulation of morphology to be separated from neurotrophin regulation of survival from the earliest stages of sensory neuron development. Growth of sensory axons on a favorable substratum is rudimentary in the absence of neurotrophins at developmental stages at which sensory neurons require these molecules for survival and their axons are extending rapidly toward peripheral targets in vivo. Several key features of early axon growth are regulated, including extension of a second axon, rate of elongation, and degree of fasciculation. Furthermore, neurotrophins support the appearance of distinct axonal morphologies that characterize different sensory neuron subpopulations.

\section{BAX levels and axon growth}

It is important at the outset to consider the possibility that our results are in some way explained by the direct involvement of BAX in signal transduction pathways that control axon outgrowth. Indeed, substantial effects of reduced levels of the BAX homolog BCL-2 on the rate of growth of sensory axons in vitro have been reported (Hilton et al., 1997). Furthermore, retinal ganglion explants from transgenic mice overexpressing BCL-2 under control of a pan-neuronal promoter extend axons more robustly than do controls at late developmental stages in vitro and in vivo after injury (Chen et al., 1997).

The relationship of BCL-2 family members to signal transduction pathways involving axon growth remains to be defined (Barde, 1997). Nevertheless, there are two compelling reasons to think that BAX itself is not crucial for axon growth and that the results reported here cannot be attributed to the absence of BAX. First, Bax-null mice develop relatively normally and survive into old age without behavioral features suggesting that axon projections or connectivity are compromised. Indeed direct counts of axons in peripheral and optic nerves of $\mathrm{Bax}-/-$ mice reveal more axons than normal, not fewer axons that would be expected if BAX itself were crucial for axon elongation (White et al., 1998).

Second, neurotrophins in standard concentrations largely reverse the abnormalities in axon growth that we have observed in Bax-null DRG neurons cultured in the absence of neurotrophins. Of note is that experiments that demonstrated effects of BCL-2 deficiency on sensory axon growth were performed in the absence of serum (Hilton et al., 1997). It is plausible that under such reduced conditions, BCL-2 levels may assume unusual importance in nonsurvival functions. Interestingly, there were no noticeable deficiencies in sympathetic ganglion neurite outgrowth when sympathetic ganglion neurons from BCL-2-deficient mice were cultured in the presence of serum and NGF (Greenlund et al., 1995).

\section{Sensory neurons are unipolar in the absence of neurotrophins}

The most striking thing about the initial inspection of cultures from the $B a x$-null mice was that virtually all sensory neurons were unipolar when cultured in the absence of neurotrophins. Both NGF and NT-3 mediated growth of a second axon, presumably from separate populations of neurons. Note that, even in the presence of neurotrophins, sensory neurons did not assume the pseudounipolar morphology that is characteristic of sensory neurons in vivo at this age. A previous study using avian sensory neurons has shown that the development of pseudounipolar morphology in vitro requires contact with Schwann cells (Mudge, 1984) that were not present in significant numbers under the culture conditions reported here.

Although it is tempting to speculate that initial development of bipolar morphology may require neurotrophin stimulation in vivo, in fact sensory neurons have already extended peripheral and central processes by E11 (Ozaki and Snider, 1997), and both were axotomized in the course of culturing these neurons. It is well established that the central process of sensory neurons in adult animals grows less well after injury than does the peripheral process (Richardson and Issa, 1984; Richardson and Verge, 1986). An intriguing possibility that would explain our results is that the difference in intrinsic potential for growth of the two 
processes after axotomy may already be present at an early stage of development and is strikingly manifest in the setting of neurotrophin deprivation. An intrinsic difference in the growth capabilities of the central versus the peripheral processes even at early developmental stages would not be surprising because peripheral sensory arborizations are obviously far more extensive than are central ones. Proof of the concept will require identification of a specific molecular marker for the central process.

\section{Neurotrophin regulation of axon elongation}

We have demonstrated that neurotrophins powerfully enhance axon growth by a mechanism that is separable from their influence on neuron survival. This issue has been controversial because previous work in chick suggested that the minimum concentration of NGF compatible with survival promoted maximum axon outgrowth at early developmental stages (Scott and Davies, 1993). We have shown here that Bax-/- sensory neurons cultured at a comparable stage in mouse extended only rudimentary axons unless neurotrophins were provided. For neurons cultured for $72 \mathrm{hr}$ starting at E13, the total axon length of Bax-null neurons was increased almost fourfold in the presence of NGF and 3.5fold in the presence of NT-3 compared with that of neurons cultured in the presence of serum but in the absence of specific neurotrophins. Importantly, the average $500 \mu \mathrm{m}$ axon length achieved over $72 \mathrm{hr}$ in the absence of neurotrophins is $<5 \%$ of the distance between the DRG and the distal hindlimb at E16, the comparable embryonic stage in vivo (Kaufman, 1992). Differences in axon length with and without neurotrophins for neurons cultured at E12 were even more impressive. The observed defect in axon fasciculation observed in less-dissociated cultures, if present in vivo, might also be expected to compromise the rate of sensory axon elongation. Our results, therefore, are consistent with the idea that neurotrophin family members are required to support the profound elongation of peripheral axons that is a concomitant of innervation of distal targets and overall growth of the embryo.

It is important to emphasize that there are two types of mechanisms, not mutually exclusive, that may underlie the effects of neurotrophins on axon elongation. It is plausible that neurotrophins may regulate mRNA levels and/or phosphorylation of cytoskeletal proteins, perhaps via the Ras/MAP kinase pathway. This type of regulation has been implicated in the NGF-induced morphological differentiation and neurite outgrowth of pheochromocytoma-12 cells (Greene and Kaplan, 1995; for review, see Kaplan and Stephens, 1994; Segal and Greenberg, 1996). It is important to point out, however, that little is known about the signal transduction pathways and genetic programs that must be activated to promote the growth of axons of primary neurons. Indeed, culture systems in which primary neurons survive in the absence of neurotrophins should become a useful tool for exploring the signal transduction pathways by which neurotrophins mediate morphological effects.

Another equally plausible mechanism of regulation of axon growth relates to the global effects of neurotrophins on mRNA and protein synthesis. Thus, sympathetic ganglion cells deprived of NGF reduce their overall protein synthesis and the levels of many mRNAs to $\sim 10 \%$ of baseline in paradigms similar to the ones shown here in which cell death is prevented by apoptosis regulators after neurotrophin deprivation (Deckwerth et al., 1998). These findings raise the intriguing possibility that general effects of neurotrophins on cell metabolism may be equally or more important in the regulation of axon growth than is the activation of specific signal transduction pathways that control the synthesis of cytoskeletal proteins.

Previous considerations of neurotrophin control of axon growth during early development have focused on chemotropic regulation over short distances and on collateral branching (for review, see Tessier-Lavigne and Placzek, 1991; McFarlane and Holt, 1997). Perhaps surprisingly in view of widespread interest, the roles of neurotrophins related to chemotropism and axon collateral branching remain undefined (Ernfors et al., 1994; O'Leary and Daston, 1994; Schimmang et al., 1995; Fagan et al., 1996; Fritzsch et al., 1997; Wright et al., 1997). Studies to date have not been definitive because of early death of the neurons in question or premature death of the animal in the absence of specific neurotrophin family members. The Bax nulls now offer a means to separate neurotrophin regulation of survival from neurotrophin regulation of axon growth in vivo. Indeed, preliminary results in mice double null for BAX and NT-3 and in mice double null for BAX and trkA show that sensory neurons survive but that the striking behavioral phenotypes associated with NT-3 or trkA deficiency are not rescued, suggesting that peripheral and/or central connections may not be established (Snider et al., 1997).

Finally, it is important to note that although neurotrophins regulate axon branching throughout life, the requirement of these molecules for axon elongation may be developmentally restricted. Indeed, dissociated sensory neurons from adult animals respond to NGF with marked branching rather than elongation (S. I. Lentz and W. D. Snider, unpublished observations; see also Smith and Skene, 1997). Furthermore, deprivation of NGF during cutaneous axon regeneration in adult animals in vivo does not affect the time course of regeneration even though NGF clearly mediates sprouting of these same axons (Diamond et al., 1992). In the PNS of adult animals, NGF and other neurotrophins, consistent with their roles as target-derived factors, typically enhance expression of genes associated with the maintenance of axon caliber such as neurofilament (Verge et al., 1990; Munson et al., 1997) and may suppress expression of genes such as GAP-43 and T $\alpha 1-\alpha$ tubulin that are normally associated with successful axon regeneration (Gratto and Verge, 1996). Our findings raise the possibility that this pattern may be reversed at early stages of neural development.

\section{Neurotrophins support the appearance of distinct axonal morphologies}

Although both NGF and NT-3 promoted growth of a second axon and axon elongation, the responses of sensitive neurons to the respective factors otherwise were quite different. Thus, unexpectedly, NGF promoted substantially greater elongation than did NT-3 and produced only modest branching that was restricted to the terminal portion of the axon. Indeed, NGF appeared to suppress branching along the main shaft of the axon. In contrast, NT-3 supported less elongation but substantially more prominent terminal branching. As demonstrated by the drawings of large numbers of neurons, the effect is robust, and there was little overlap in the appearance of neurons treated with the two factors at E13. Importantly, these distinctive responses were also present at E12. These prominent effects of NT-3 on axon branching are consistent with the findings of Erzurumlu and collaborators who have found that NT-3 promotes collateral branching of trigeminal sensory axons into the brainstem (Ulupinar and Erzurumlu, 1998). The early appearance of the characteristic NT-3 branching response suggests the presence of inhibitory molecules, possibly semaphorin family members, along the pathways of NT-3- 
responsive neurons that may suppress axon branching (Taniguchi et al., 1997).

BDNF produced morphological responses similar to that of NT-3 in approximately the same percentage of neurons at E12, suggesting that there is substantial overlap in the sensory subpopulations responding to these two factors in early development. Indeed recent findings have shown extensive coexpression of trkB and trkC at E11 and dependence of sensory neurons expressing both receptors on NT-3 (Fariñas et al., 1998). In addition, BDNF induced large and complex lamellipodia along the distal axon shafts from a subset of its responsive neurons. Perhaps surprisingly, in view of clear-cut morphological effects induced by BDNF, most investigators have not found that BDNF supports the survival of a significant percentage of embryonic DRG neurons in vitro, and whether there is an in vivo survival requirement of DRG neurons for BDNF is controversial (see Matheson et al., 1997, references therein; Silos-Santiago et al., 1997, references therein).

These differing morphological responses could be interpreted as evidence of an "instructive" role for neurotrophins in shaping sensory axon morphology. Much current evidence is consistent with the idea that characteristic axonal and dendritic morphologies throughout the nervous system could be, in part, determined by local patterns of neurotrophin expression and/or spatial segregation of trk expression by responsive neurons (Segal et al., 1995; Neveu and Arenas, 1996; McAllister et al., 1997). Indeed, our demonstration of pronounced regulation of branching by NT-3 may explain recently reported results of differing effects of transgenic overexpression of NGF and NT-3 on arborizations of trigeminal sensory axons in the mystacial pad in vivo (Davis et al., 1997; Rice et al., 1998; see also ElShamy et al., 1996). The concept of instructive effects would predict that transfection of the "wrong" trk into a particular sensory subpopulation would convert its axonal morphology.

Of particular note in the results reported here, however, is that NGF- and NT-3-responsive neurons almost certainly represent different populations. In this situation, neurotrophins may also have a "permissive" role in allowing the appearance of characteristic morphologies specified at an early stage of differentiation. Thus, in addition to inducing a particular type of morphological response, neurotrophins may also regulate the expression of receptors and surface molecules necessary for intrinsic differences in cytoskeletal organization to become manifest and for axons to respond in a distinctive manner to local cues (e.g., Tuttle and O'Leary, 1998). Viewed in this context, NT-3-dependent (primarily proprioceptive) and NGF-dependent (primarily nociceptive) neurons may have intrinsic capabilities for differing axonal morphologies, presumably analogous to better-characterized differences in dendritic morphologies among different neuronal classes. Such a permissive role for neurotrophins seems likely to be widespread because this family that has only four known members in mammals regulates neurons throughout the PNS and CNS.

\section{REFERENCES}

Barde Y-A (1997) Help from within damaged axons. Nature 385:391-393.

Buchman VL, Davies AM (1993) Different neurotrophins are expressed and act in a developmental sequence to promote the survival of embryonic sensory neurons. Development 118:989-1001.

Chen DF, Schneider GE, Martinou J-C, Tonegawa S (1997) Bcl-2 promotes regeneration of severed axons in mammalian CNS. Nature 385:434-439.

Davies AM, Bandtlow C, Heumann R, Korsching S, Rohrer H, Thoenen
H (1987) Timing and site of nerve growth factor synthesis in developing skin in relation to innervation and expression of the receptor. Nature 326:353-358.

Davis BM, Fundin BT, Albers KM, Goodness TP, Cronk KM, Rice FL (1997) Overexpression of nerve growth factor in skin causes preferential increases among innervation to specific sensory targets. J Comp Neurol 387:489-506.

Deckwerth TL, Elliott JL, Knudson CM, Johnson Jr EM, Snider WD, Korsmeyer SJ (1996) Bax is required for neuronal death after trophic factor deprivation and during development. Neuron 17:401-411.

Deckwerth TL, Easton RM, Knudson CM, Korsmeyer SJ, Johnson Jr EM (1998) Placement of the BCL-2 family member BAX in the death pathway of sympathetic neurons activated by trophic factor depravation. Exp Neurol 152:150-162.

Diamond J, Foerster A, Holmes M, Coughlin M (1992) Sensory nerves in adult rats regenerate and restore sensory function to the skin independently of endogenous NGF. J Neurosci 12:1467-1476.

Eichler ME, Rich KM (1989) Death of sensory ganglion neurons after acute withdrawal of nerve growth factor in dissociated cell cultures. Brain Res 482:340-346.

ElShamy WM, Linnarsson S, Lee KF, Jaenisch R, Ernfors P (1996) Prenatal and postnatal requirements of NT-3 for sympathetic neuroblast survival and innervation of specific targets. Development 122:491-500.

Ernfors P, Merlio J-P, Persson H (1992) Cells expressing mRNA for neurotrophins and their receptors during embryonic rat development. Eur J Neurosci 4:1140-1158.

Ernfors P, Lee KF, Jaenisch R (1994) Mice lacking brain-derived neurotrophic factor develop with sensory deficits. Nature 368:147-150.

Ernfors P, Van De Water T, Loring J, Jaenisch R (1995) Complementary roles of BDNF and NT-3 in vestibular and auditory development. Neuron 14:1153-1164.

Fagan AM, Zhang H, Landis S, Smeyne RJ, Silos-Santiago I, Barbacid M (1996) TrkA, but not trkC, receptors are essential for survival of sympathetic neurons in vivo. J Neurosci 16:6208-6218.

Fariñas I, Yoshida CK, Backus C, Reichardt LF (1996) Lack of neurotrophin-3 results in death of spinal sensory neurons and premature differentiation of their precursors. Neuron 17:1065-1078.

Fariñas I, Wilkinson GA, Backus C, Reichardt LF, Patapoutian A (1998) Characterization of neurotrophin and Trk receptor functions in developing sensory ganglia: direct NT-3 activation of TrkB neurons in vivo. Neuron 21:325-334.

Fritzsch B, Silos-Santiago I, Bianchi LM, Fariñas I (1997) The role of neurotrophic factors in regulating the development of inner ear innervation. Trends Neurosci 20:159-164.

Gratto KA, Verge VMK (1996) The role of NT-3 in the regulation of GAP-43 and t $\alpha 1$-tubulin in intact and injured primary sensory neurons. Soc Neurosci Abstr 22:1001.

Greene LA, Kaplan DR (1995) Early events in neurotrophin signalling via trk and p75 receptors. Curr Opin Neurobiol 5:579-587.

Greenlund LJS, Korsmeyer SJ, Johnson Jr EM (1995) Role of BCL-2 in the survival and function of developing and mature sympathetic neurons. Neuron 15:649-661.

Hilton M, Middleton G, Davies AM (1997) Bcl-2 influences axonal growth rate in embryonic sensory neurons. Curr Biol 7:798-800.

Johnson Jr EM, Deckwerth TL, Deshmukh M (1996) Neuronal death in developmental models: possible implications in neuropathology. Brain Pathol 6:397-409.

Kaplan DR, Stephens RM (1994) Neurotrophin signal transduction by the trk receptor. J Neurobiol 25:1404-1417.

Kaufman MH (1992) The atlas of mouse development. San Diego: Academic.

Lumsden AGS, Davies AM (1983) Earliest sensory nerve fibres are guided to peripheral targets by attractants other than nerve growth factor. Nature 306:786-788.

Matheson CR, Carnahan J, Urich JL, Bocangel D, Zhang TJ, Yan Q (1997) Glial cell line-derived neurotrophic factor (GDNF) is a neurotrophic factor for sensory neurons: comparison with the effects of the neurotrophins. J Neurobiol 32:22-32.

McAllister AK, Katz LC, Lo DC (1997) Opposing roles for endogenous BDNF and NT-3 in regulating cortical dendritic growth. Neuron 18:767-778.

McFarlane S, Holt CE (1997) Growth factors: a role in guiding axons? Trends Cell Biol 7:424-430.

McFarlane S, McNeill L, Holt CE (1995) FGF signaling and target 
recognition in the developing Xenopus visual system. Neuron 15:1017-1028.

Mudge AW (1984) Schwann cells induce morphological transformation of sensory neurones in vitro. Nature 309:367-369.

Munson JB, Shelton DL, McMahon SB (1997) Adult mammalian sensory and motor neurons: roles of endogenous neurotrophins and rescue by exogenous neurotrophins after axotomy. J Neurosci 17:470-476.

Neveu I, Arenas E (1996) Neurotrophins promote the survival and development of neurons in the cerebellum of hypothyroid rats in vivo. J Cell Biol 133:631-646.

O'Leary DD, Daston MM (1994) Neurotrophin-3 has a chemotrophic effect on cortical neurons. Soc Neurosci Abstr 20:1685.

Ozaki S, Snider WD (1997) Initial trajectories of sensory axons toward laminar targets in the developing mouse spinal cord. J Comp Neurol 380:215-229.

Rice FL, Albers KM, Davis BM, Silos-Santiago I, Wilkinson GA, LeMaster AM, Ernfors P, Smeyne RJ, Aldskogius H, Phillips HS, Barbacid M, DeChiara TM, Yancopoulos GD, Fundin BT (1998) Differential dependency of unmyelinated and A $\delta$ epidermal and upper dermal innervation on neurotrophin trk receptors and p75LNGFR. Dev Biol 198:57-81.

Richardson PM, Issa VMK (1984) Peripheral injury enhances central regeneration of primary sensory neurons. Nature 309:791-793.

Richardson PM, Verge VMK (1986) The induction of a regenerative propensity in sensory neurons following peripheral axonal injury. J Neurocytol 15:585-594.

Schimmang T, Minichiello L, Vazquez E, San Jose I, Giraldez F, Klein R, Represa J (1995) Developing inner ear sensory neurons require trkB and trkC receptors for innervation of their peripheral targets. Development 121:3381-3391.

Scott SA, Davies AM (1993) Age-related effects of nerve growth factor on the morphology of embryonic sensory neurons in vitro. J Comp Neurol 337:277-285.

Segal RA, Greenberg ME (1996) Intracellular signaling pathways activated by neurotrophic factors. Annu Rev Neurosci 19:463-489.

Segal RA, Pomeroy SL, Stiles CD (1995) Axonal growth and fasciculation linked to differential expression of BDNF and NT3 receptors in developing cerebellar granule cells. J Neurosci 15:4970-4981.

Silos-Santiago I, Fagan AM, Garber M, Fritzsch B, Barbacid M (1997) Severe sensory deficits but normal CNS development in newborn mice lacking trkB and trkC tyrosine protein kinase receptors. Eur J Neurosci 9:2045-2056.

Smith DS, Skene JHP (1997) A transcription-dependent switch controls competence of adult neurons for distinct modes of axon growth. J Neurosci 17:646-658.

Snider WD, White FA, Molliver DC, Knudson CM, Korsmeyer SJ (1997) Elimination of BAX fails to rescue the NT-3 null mutant phenotype. Soc Neurosci Abstr 23:617.

Taniguchi M, Yuasa S, Fujisawa H, Naruse I, Saga S, Mishina M, Yagi T (1997) Disruption of semaphorin III/D gene causes severe abnormality in peripheral nerve projection. Neuron 19:519-530.

Tessier-Lavigne M, Placzek M (1991) Target attraction: are developing axons guided by chemotropism? Trends Neurosci 14:303-310.

Tuttle R, O'Leary DDM (1998) Neurotrophins rapidly modulate growth cone response to the axon guidance molecule, collapsin-1. Mol Cell Neurosci 11:1-8.

Ulupinar E, Erzurumlu R (1998) Effects of neurotrophins on axon growth patterns in explant cultures of the central trigeminal pathway. Soc Neurosci Abstr 24:27.

Verdi JM, Groves AK, Fariñas I, Jones K, Marchionni MA, Reichardt LF, Anderson DJ (1996) A reciprocal cell-cell interaction mediated by NT-3 and neuregulins controls the early survival and development of sympathetic neuroblasts. Neuron 16:515-527.

Verge VMK, Tetzlaff W, Bisby MA, Richardson PM (1990) Influence of nerve growth factor on neurofilament gene expression in mature primary sensory neurons. J Neurosci 10:2018-2025.

White FA, Silos-Santiago I, Molliver DC, Nishimura M, Phillips H, Barbacid M, Snider WD (1996) Synchronous onset of NGF and trkA survival dependence in developing dorsal root ganglia. J Neurosci 16:4662-4672.

White FA, Keller-Peck CR, Knudson CM, Korsmeyer SJ, Snider WD (1998) Widespread elimination of naturally occurring neuronal death in Bax-deficient mice. J Neurosci 18:1428-1439.

Wilkinson GA, Fariñas I, Backus C, Yoshida CK, Reichardt LF (1996) Neurotrophin-3 is a survival factor in vivo for early mouse trigeminal neurons. J Neurosci 16:7661-7669.

Wright DE, Zhou L, Kucera J, Snider WD (1997) Introduction of a neurotrophin-3 transgene into muscle selectively rescues proprioceptive neurons in mice lacking endogenous neurotrophin-3. Neuron 19: 503-517. 